\title{
Human Systems Biology
}

\author{
Bhrugesh P. Joshi ${ }^{1 *}$, Roshni K. Bhatt ${ }^{2}$ and DR. R. Krishnamurthy ${ }^{3}$ \\ 1, 2\&3 (C. G. Bhakta Institute of Biotechnology, Bardoli Mahuva Road, Dis.: Surat - 394 350, Gujarat, INDIA)
}

\begin{abstract}
Human Systems Biology (HSB) is the pertinence of computational biology to Human health and disease, with scrupulous emphasis on the contrive of cellular, molecular and structural biology. HSB is a relatively new field of network analysis and systems biology. That focuses on the, high quality databases, the analyses of data that are generated in systems biology and network medicine approaches and the generation of testable hypothesis. HSB originally emerged as an ameliorate the drug discovery and development process. However HSB now plays an elaborating paramount role in many areas of proteomics, genomics, metabonomics biophysics and biomedical engineering. HSB can also be applied to new drug invention for pharmaceutical industries.
\end{abstract}

Keywords: Human disease, New drug application, Whole organ modeling.

\section{INTRODUCTION}

HSB is the use of computational biology and human biology, applied to an ambit of complication in human health and diseases. Also known as HSB. These fields are used in pharmaceutical industries in the process of new drug discovery, personalized medicine, translating the systems biology framework to the human systems. HSB combines the scientific working field of human biological science and computer science exceedingly in the area of molecular modeling or structural biology and sequence analysis it is to be expected that the simulation of molecular network, probabilistic data integration, and development of drug cocktails. In quantitative techniques, large-scale measurement methods and with the intimate integration between experimental and computational approaches, human biological science has contemporary acquired new technological and ideational tools to interrogate, model and understanding living organism at the systems level, to study well- characterized model organism. That appliance of system-wide resemble to human biology would open up formidable contingency in medicine.

\section{ROLE OF MEDICAL RESEARCH IN HSB}

In a research area in medical science, comprise oncology and oncoproteomics, construe the mechanisms of disease requires a deep lore of how signaling transduction pathways amputated. Quantitative proteomics has made credible the coetaneous cover of the circumstantial exertion of conjoint signaling molecules, implementing a broader and detached view of cellular signaling events. The work by White and coworkers [1] illustrates how this type of high-throughput data can be supplemented to biological response (e.g., proliferation and cell migration) to further our understanding of one of the major pathways known to be deregulated in cancer. These global approaches also reveal the forthcoming fact that biological pathways are highly concatenate, which portray one of the major motivations for espouse a system-level approach in human biological science. That by [2] the impact of interconnectivity on biological circuit outcome is anatomize to demystify synergies and non- intuiting other dealings apparent between simultaneously applied drugs, with important consequences for computer aided drug design and pharmacogenomics.

The innovation in molecular biology, conspicuous in the Human Genome Project, has compulsive and has been driven by technological advances that have simplified the concurrent amassment of a large number of biological parameters. Examples include microchip arrays that employ antibody fragments for detection and measurement of thousands of gene products (mRNA) in a single assay and the development of similar chips for application with protein molecules. More established techniques, such as mass spectrometry, have made commensurate improvement and are now capable of measuring molecules of higher mass with greater throughput. These overtures are helping the provision of ever more quantitative information about biological systems at different spatial and temporal levels and can be employed to make more useful and predictive models.

Large-scale integrated models of disease, such as diabetes and obesity, are being entrenched for the simulation of the clinical effects resulting from manipulations of one or more drug targets. These models will facilitate the selection of the most relevant targets and help in planning clinical trials. Coupling this approach with pertinent genomic information holds the promise of analyzing patients likely to benefit most from or to be harmed by, a particular therapy as well as helping in the lamination of patients in clinical trials. 
Biomarkers are biological indicators that are used to assess the biological state of an organism, the advancement of a disease or the effect of treatments. Blood pressure and heart rate, for instance, are simple indicators of cardiovascular function. New and more complex biomarkers include biological molecules such as the tumour specific carcino-embryonic antigen that can be used to track progression of colon cancer. In some cases, multiple biomarkers are used in liability easement. For example blood pressure, LDL cholesterol, blood sugars predict the liability of a myocardial infarction. There is currently enormous concern in the probable of new indicators that have been invented using methods such as gene expression and proteomics. However, the clarification of their implication will have to take account of physiological and pathophysiological changeability and the extent to which they are linked to disease mechanism. In many cases, patterns of changes in a number of biomarkers, rather than the biomarkers themselves, whose role may hover unknown, are used as biological indicators. In order to use biomarkers to their enormous response, a correct definition of their reading and their appropriate capability will be imperative and human systems biology has the potential to provide the right tools to do so.

\subsection{Oncology}

Is a molecular disease that associates mutations (usually more than one) in genes that control cellular division or death? Molecular biology is having amazing success in defining the exact mutations that may, for example, cause undisciplined cellular division and growth. Hence, the treatment of cancer is emerge away from the conventional method of using highly toxic drugs in superlative stand for doses towards an access that is highly targeted to specific defects. A timely example is provided by the use of the antibody Herceptin to treat breast cancer indicated by the over-expression of the HER2 protein. Tumours can be effective at eluding Interventions aimed at destroying them. For instance, some cancers express factors that dislodge drugs from the tumour cells, thus avert their action. However, cancer cells need a blood supply to divide and grow and this need is being exploited by specific drugs that constrain the growth of blood vessels into tumours. Treatment of cancers must be monitored and imaging technologies are key in assessing the response of tumours to treatment. In particular, methods that measure blood flow or metabolism are giving much earlier assessment of tumour response than the standard way of measuring tumour size. The tracking and the modelling of factors that influence drug delivery and penetration, and tumour response are critical to the understanding of cancer mechanisms and their treatment.

\subsection{Infectious diseases}

The escalation of infections and the ability of pathogens to expand scenario that reduce or defeat host defenses against them are issues that application a systems approach. Knowledge of the means by which organisms exploit weaknesses in host defense systems is increasing rapidly. Some viruses (e.g. influenza, HIV) take advantage of rapid mutation to evade immune surveillance or drug action. The Epstein Barr virus (infectious mononucleosis and Burkitt's lymphoma), tuberculosis bacterium, malaria parasite and even some strains of streptococci are all examples of common pathogens that have developed strategies to evade or neutralize host defense. The complication of dealing with these infections is affidavit to the gravity of the clinical problem that pathogens construct. Studies of infections require a consolidation of knowledge of the pathogen, the host, the environment and the available treatments. It will only be desirable to maintain such revenue of information using a systems approach. Problems of hospital-cultivate infections such as MRSA or Clostridium difficile, for example, are suitable subjects for a systems-based enquiry.

Epidemiology makes all inclusive use of predictive modelling. Indeed, one paramount utilization of HSB in the public health stage is through complex stochastic models that have been developed to support planning for the control of a novel influenza A, the agent responsible for flu pandemics. Such models use 'individuals-based' simulation approaches. Computational problems such as this are very complex and require novel interdisciplinary approaches to blending biological, clinical, epidemiological, demographic and behavioral data. They can therefore accountable from the tools those HSB can provide.

\section{WHOLE-ORGAN MODELING}

An exhaustive bottom-up reformation of a complex organ such as the heart would be very crucial. Therefore, when modelling higher level physiological systems, activity at lower spatial levels is illustrated with simplified equations. The engineering principle of modularity discussed earlier in this chapter can be abuse so that sub-systems within a larger model are represented by 'black boxes', mutually with feedback and feed forward loops, where the input and output, but not the intervening steps, are considered. Ultimately, approach in HSB will also allow the detailed compassionate of the function of these black box modules. Such modularity permits the adoption of a middle-out, rather than bottom-up or top-down, approach. When choosing the middleout option, analysis starts at the level for which there are large amounts of usable data and then reaches out in either direction (this is the 'out' part of the metaphor) to consider the next linked modular subsystem. All three 
approaches are needed, although the general view is that top-down and middle-out approaches are likely to be more profitable in addressing future therapeutic needs, at least for the near-term future. Modularity also enables the identification of relevant features of the lower-level mechanisms. Foremost, this allows the complexity of the overall model to be kept within bounds; for example, sodium or calcium transport can be described by a few simple equations. It is important to note that incomplete or proximate models can have significant value and application, and it is not necessary to wait for all the details of a system to be defined before a model can be used. Indeed, the outcome of a model can be used in combination with experimental data for further clarification of the model itself through the cycle of iteration between modelling and experimentation described earlier. The results of modelling complex systems are again and again counterintuitive. Beyond a certain degree of complexity, qualitative thinking is not only inadequate, it can even be inaccurate. A good example of this is provided by the mechanism of mechano-electric feedback, in which the contraction of the heart influences its electrical properties. Some of the results, particularly on the actions of changes in cell volume (characteristics of many disease states), are unexpected and have been responsible for determining the next stage in experimental work. The resolve of such complex physiological processes can only occur as a result of the iterative exchange between experiment and simulation.

\subsection{Victorious information of HSB}

HSB is accrual furnishing to the understanding of medical conditions and the way these respond to treatments. For instance, the US Company Entelos Inc. has developed functional computer models of diabetes, obesity, rheumatoid arthritis and asthma that are being used in the design of clinical trials

As part of the Physiome Project, a model of the heart has also been developed to assess the risk of 'torsade de pointes' (a potentially fatal cardiac arrhythmia that can be provoked by rare inherited conditions affecting an ion channel in the heart) and test some therapeutic drugs that prolong the repolarization of the heart muscle by inhibiting the function of this channel. The model has already been used successfully in the impositions of drug safety by regulatory bodies in the US and Europe. Another successful model has been developed to assess the determinants of the response to COX inhibitors, an analgesic class of drugs. There is a moderately destitute supplementation between the blood plasma concentration of this class of drug and the analgaesic or adverse effects in chronic inflammatory conditions. Thereupon, it is difficult to predict the appropriate dose regimes for the treatment of chronic inflammatory pain. The modelling of changes in endogenous mediators of inflammation has helped to elucidate the relation between exposure to the drug and the therapeutic response. HSB, For instance, the Physiome Project seeks to accomplish a quantitative description of the whole human organism, while the Receptor Tyrosine Kinase (RTK) Consortium strives to facilitate and coordinate international efforts for the understanding of RTK signaling pathways and their relationship to human pathologies.

\subsection{Computational biology strategies for new drug discover}

Molecular modeling or structural biology is the most deep-rooted bailiwick of computational biology. It aims to predict and model parcel of biological targets, and rests on the shoulders of chemistry, quantum and biophysics, experimental crystallography and computer science. Alongside established tools of computational chemistry, which model properties of drug leads and their interaction with targets, molecular models form the centerpiece of computational drug discovery and development. Sequence analysis aims to efficiently compare nucleotide and amino acid sequences, thereby allowing researchers to impute a gene's function by regarding clue from homologous genes, often from different biological systems. Sequence analysis has now become an imperative part of target identification in early-stage drug discovery

$>\quad$ Imaging refashioning in disease.

$>$ Computer models to prognosticate drug action.

$>$ New blistering approaches to detecting diseases.

$>$ Computer models to detect toxicity.

$>$ Biochemistry in silico.

$>$ Seeing genes in action.

$>\quad$ More effective therapeutics that tackles the underlying causes of disease rather than treating the symptoms - pharmaceuticals with affection side effects.

$>\quad$ Providing bio-industry with the competence to model and exploit biological processes better so as to provide unheard compounds for the chemical, pharmaceutical and thereby improving the competitive acridity of these industries.

$>\quad$ A exceed understanding of healthy ageing and how to keep up a population that abide healthy and constructive for longer.

$>\quad$ The elaboration of predictive (in silico) toxicology models of cells and organs leading to extemporization drug screens and downgrade lack for animal testing. 


\section{HSB AND NETWORK ANALYSIS IN HUMAN BIOLOGICAL SYSTEMS}

The classical generality of linear pathways is being increasingly abled by network analysis, which foregrounds the importance of interactions between components of a biological system. [3] Discuss how this network-based conceptual architecture may transform current paradigms in disease prescriptive and treatment. However, a major actionable challenge is how to presume the structure of complex networks that fundamental biological procedure and how to depict their state when concern by disease. New computational strategies like's omics-databases in mainly newtomics mingled with the now entrenched genome-wide expression profiling techniques provide entirely new tools to simulation network structure and to identify and track mediators cohort with a disease as illustrated by the work of Jim Collins' team on prostate cancer [4]. Progression of the field of metabonomics, earliest by Jeremy Nicholson. His study reveals the abstruse influence exerted by gut bacterial flora on the metabolic equilibrium of the host and, as a consequence, on its health status. This work also prove that the genotype-phenotype kinship is far from being the exclusive story when dealing with disease, and it highlights the critical importance of assimilate all facets of physiology, including benefaction from the microbiome and environment, thus adopting an even wider ambit than the genome-wide paradigm. The gigantic hopes generated by the usance of high-throughput technologies like in omics research the interference between large scale biology such likes genome, proteome, transcriptome. To human samples is that the elevated amount of admonition gathered can facade to more robust models able to anticipate vulnerability to disease, answer to treatment and, conceivably even more challenging, help in the prognosis of disease aftereffect. It is the latter question of prognosis that is declamation in the study by MacBeath and co-workers [5], where assimilation of clinical parameters with protein microarray magnitude of blood samples allows augmented prediction of early mortality of patients initiating a kidney dialysis treatment. Progressive application of these technologies is likely to be instrumental in opening the door to the epoch of personalized medicine with perfectly strategies circumferential all dimensions of clinical practice, including prevention, diagnosis, treatment and prognosis.

The firsthand usage of Systems Avenue to clinical medicine. Significant resembling subsists between progressive imaging and HSB in that both necessitate an amalgamation of mathematical, statistical and biological training. Neuroscience, for instance, is an area where close relations between biologists and imaging scientists will be essential and where HSB will play a significant role in translational/experimental medicine.

Translating the HSB framework to the human biological system will emblematize a terrific challenge, not only because of the appalling complexity of human physiology, but also because the human condition evidence deliberate scrutiny of ethical, legal, safety, individual and epidemiological issues. Revolutionary technologies, novel insights, colossal digitalization of information will call for clear thinking and innovation in the formulation of governance policies. It is thus also our hope that by providing a portion of some of the recent concrete significant addition to the tract, we can stimulate, already at this early stage, reflections and controversy, extending beyond the HSB community, so that the full potential and promises of Systems Medicine can be actualized in harmony with societal standards.

\section{CONCLUSION}

HSB on healthcare will be consequential. By that time, it is likely that modelling of human disease will be the accepted norm rather than the demurrer. This will lead to a routine understanding of the physiological mechanisms that are disintegrated in the pathogenesis of complex diseases and the development of therapies whose effects are understood in the context of the whole organism rather than in isolated assays. Synthetic Biology will have developed adequately for its demesne of application to have become unquestionable defined and for business to invest securely in the field. Areas such as chemical engineering, biomaterials, electronics and computing will be undergoing significant makeover. Multidisciplinary science will be prosaic, with biologists, engineers and mathematicians all able to transmit 'in the same language'. During this time, there will have been a clear move from team working by scientists trained in single province to teams of scientists from both biological and physical disciplines working together on specific projects. In order to address the challenges of human health through the delivery of novel therapies, academia and industry will have evolved mechanisms of working together to develop and apply new science more effectively. By this time, it is likely that HSB will have become another tool to be applied experimentally, much as molecular biology is today, and will not demand the existence of large, complex organizational infrastructures. It is also probable that clinical support systems that use HSB equipment will have become a routine part of improving care of patients affected by multiple conditions who require treatment with a wide array of possible diagnostic and therapeutic alternatives.

Acknowledgements

We all authors are to be the very thankful institution of CGBIBT. Almighty embark of knowledge. 


\section{REFERENCES}

[1] Wolf-Yadlin A, Kumar N, Zhang Y, Hautaniemi S, Zaman M, Kim HD, Grantcharova V, Lauffenburger DA, White FM Effects of HER2 overexpression on cell signaling networks governing proliferation and migration. Molecular Systems Biology, 2, $2006,54$.

[2] Leha'r J, Zimmermann GR, Krueger AS, Molnar RA, Ledell JT, Heilbut AM, Short GF, Giusti LC, Nolan GP, Magid OA, Lee MS, Borisy AA, Stockwell BR, Keith CT Chemical combination effects predict connectivity in biological systems. Molecular Systems Biology, 3, 2007, 80

[3] Loscalzo J, Isaac Kohane I, Barabasi AL Human disease classification in the postgenomic era: a complex systems approach to human pathobiology. Molecular Systems Biology, 3, 2007,124.

[4] Ergu"n A, Lawrence CA, Kohanski MA, Brennan TA, Collins JJ A network biology approach to prostate cancer. Molecular Systems Biology, 3, 2007, 82 .

[5] Knickerbocker T, Chen JR, Thadhani R, MacBeath G An integrated approach to prognosis using protein microarrays and nonparametric methods. Molecular Systems Biology, 3, 2007, 123.

[6] Butcher E Can cell systems biology rescue drug discovery? Nature Reviews Drug Discovery, 4(6), 2005, 461-67.

[7] Hunter P, Robbins P \& Noble D The IUPS human physiome project. European Journal of Physiology, 445, $2002,1-9$.

[8] Huntjens DR, Danhof M \& Della Pasqua OE Pharmacokinetic-pharmacodynamic correlations and biomarkers in the development of COX-2 inhibitors. Rheumatology, 44(7) 2005, 846-59.

[9] Kitano H Systems biology: a brief overview. Science, 295, 2002, 1662-64.

[10] Westerhoff HV \& Palsson B (2004). The evolution of molecular biology into systems biology. Nature Biotechnology, 22, 2004, $1249-52$

[11] Lixia Yao James A. Evans and Andrey Rzhetsky Novel opportunities for computational biology and sociology in drug discovery. Trends Biotechnol, 27(9), 2009, 531-540.

[12] WWW.ENTELOS.COM (RHEUMATOID ARTHRITIS MODELING)

[13] www.physiomeproject.org 